\title{
Preliminary genetic characterization of Felis Catus; European shorthair cat from Bosnia and Herzegovina
}

\author{
Esma Zajimović ${ }^{1}$, Jasmin Šutković ${ }^{2^{*}}$ \\ ${ }^{1,2}$ Genetics and Bioengineering department, International University of Sarajevo (IUS). \\ ${ }^{*}$ Corresponding author: jsutkovic@ius.edu.ba
}

Abstract
Abrresponding author: jsutkovic@ius.edu.

(C) The Author 2020.

Published by

ARDA.

\begin{abstract}
Felis Catus is a small carnivorous mammal and it is considered to be the only domesticated species among Felidae family. The purpose of this work is to genetically characterize cat breeds from Bosnia and Herzegovina and to compare them to one unknown completely different cat. To achieve this, samples of 20 cats that belong to the European Shorthair Cat (ESH) breed have been collected, plus the target subject. Further, for the genetic microsatellite characterization, the DNA material was isolated from each cat, in order to compare them to the sample taken from an unknown cat breed that will be referred to as the subject of this research. Genetic diversities within and between populations were be analyzed using 5 microsatellite markers. The obtained results showed that the subject cat genetically differs from other ESH breed cats, where the observed heterozygosity patterns within the cat breeds showed minimum but expected genetic variety among the analyzed cat species.
\end{abstract}

Keywords: Microsatellites; ESH; Genetic diversity, Phylogeny, Cats

\section{Introduction}

Felis Catus is a small carnivorous mammal cat and it is the only domesticated species among Felidae. The most abundant breed of domestic cat in West Balkan is European Shorthair cat (ESH) [1]. On the contrary ESHs are rare in United States and are rarely found. ESH cats are known to be one of the oldest cat breeds of Continental Europe. The ESH is not recognized by any of U.S. cat clubs but is accepted by Fédération Internationale Féline (FIFe) [2]. They can be either kept in house as pets or be feral, living freely, keeping distance from humans. It is impossible to predict the temperament of a European Shorthair. Each cat must be judged individually [1]. History of European Shorthair Cat goes back to time of Rome Emperors. As the Roman legions were marching throughout Europe to spread their culture, they have domesticated cats for the purpose of protecting their food from mice and rats, because cats are really good hunters and rodents are their natural prey [3]. Throughout this historic purpose and role importance for Romans, this breed is referred to as Roman cat. ESH cats tend to be bicolored, with a short-haired tabby mantle but may also carry long haired, hawkish or marbled genes as shown on the Figure 1[4]. This study represents an initial and preliminary genetic characterization on Felis Catus varieties, found on Bosnian and Herzegovina land. We tried to genetically correlate domestic cat species, for their evolutionary inheritance through phylogenetic analysis. For this purpose, we used several microsatellite genetic markers. This study involved 20 different cats, all ESH breeds, as shown in the Table 1. The genetic material of these cats was isolated in order to compare it to sample taken from unknown cat breed, that will be further in the text referred to as a Subject of this research. 


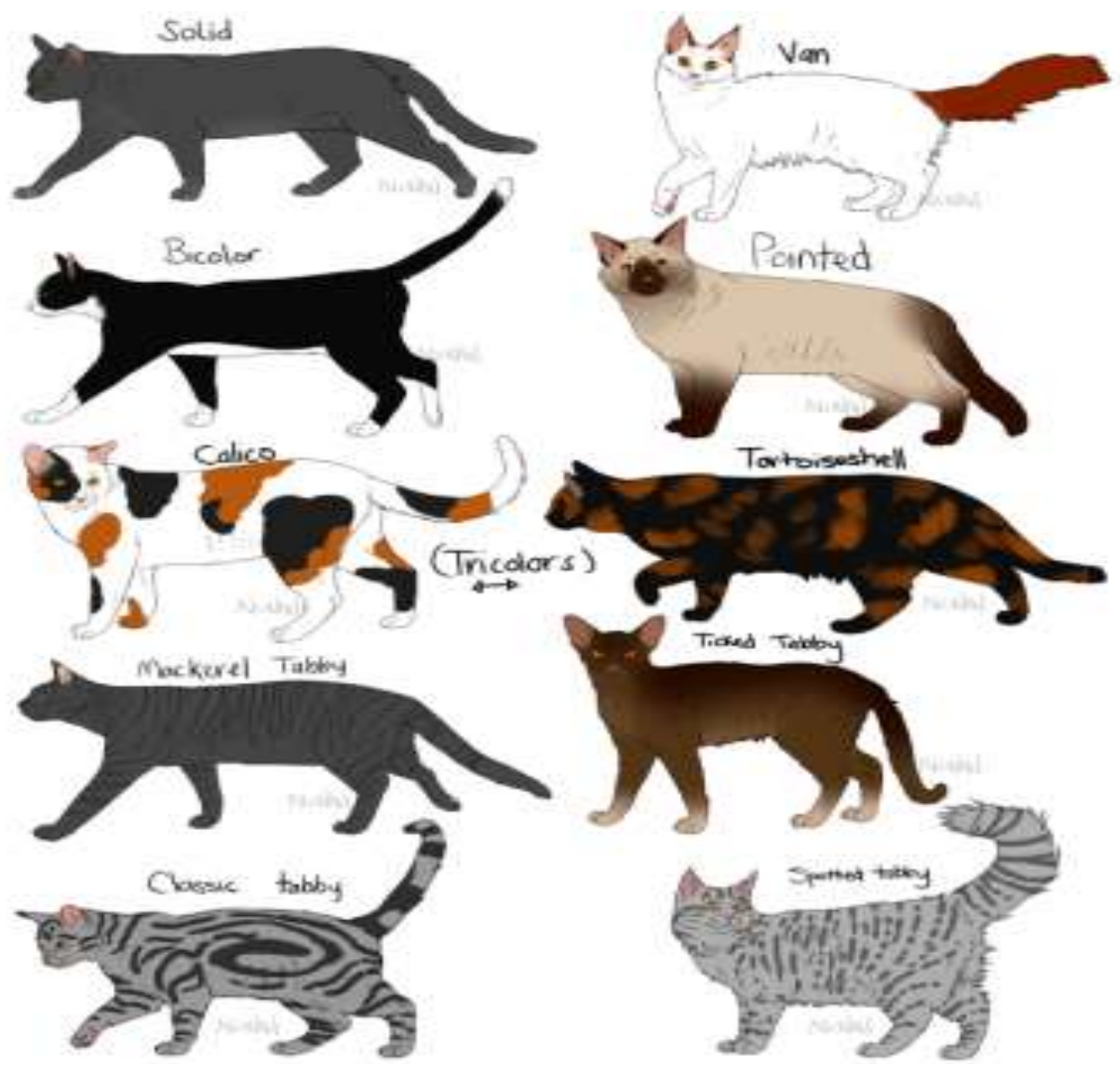

Figure 1. The most conserved cat coat patterns among ESH [4].

\section{Material and Methods}

In table 1 the cat varieties used in this study are shown. All varieties analyses and sample taken from cities in Bosnia and Herzegovina.

Table 1: Cats analyzed throughout this research

\begin{tabular}{|l|l|l|l|l|l|}
\hline No & Name of cat & Gender & Age & Habitat & Coat color \\
\hline 1 & Mišur & Female & 6 months & Mostar & Bicolor \\
\hline 2 & Lazo & Male & 6 months & Mostar & Bicolor \\
\hline 3 & Maša & Female & 12 months & Mostar & Solid \\
\hline 4 & Cuki & Male & 7 months & O. Brijeg & Tabby \\
\hline 5 & Vitany & Female & 18 months & O. Brijeg & Tabby \\
\hline 6 & Merdanova & Female & 12 months & O. Brijeg & Bicolor \\
\hline 7 a & Cuni & Male & 24 months & Tešanj & Solid \\
\hline 8 & Tipka & Female & 24 months & Tešanj & Calico \\
\hline 9 & Pipi & Female & 18 months & Tešanj & Calico \\
\hline 10 & Crni & Male & 24 months & Tešanj & Solid \\
\hline 11 & Žuti & Male & 24 months & Tešanj & Tabby \\
\hline 12 & Sivii & Male & 36 months & Tešanj & Tabby \\
\hline 13 & Žućo & Male & 6 months & Tešanj & Tabby \\
\hline 14 & Crna & female & 8 months & Tešanj & Solid \\
\hline 15 & Mimica & female & 3 months & Tešanj & Calico \\
\hline 16 & Subject & male & 24 months & O. Brijeg & Solid \\
\hline 17 & Unknown & female & 18 months & O. Brijeg & Solid \\
\hline 18 & Sarabi & female & 4 months & Visoko & Solid \\
\hline 19 & Rudi & male & 6 months & Tešanj & Paint \\
\hline 20 & Unknown & male & 24 months & O. Brijeg & Tabby \\
\hline
\end{tabular}




\subsection{Genomic DNA isolation and quantification}

DNA samples were collected by gently rubbing inside of cat's mouth using non-invasive cotton tipped swab. We strictly adhered to rules of Ethics in animal use [5]. The DNA was extracted according the modified Kern Lysis isolation protocol (Kern lysis buffer, $5 \mathrm{x}$ TBE buffer, 20\%SDS and $6 \mathrm{M} \mathrm{NaCl}$ ), using Proteinase K [6]. The DNA quality was analyzed on $1 \%$ agarose gel and quantified using a Multiscan GO (Thermo Fisher Scientific, USA), using a $\mu$ Drop plate, and stored in $100 \mu$ aliquots in $1 \mathrm{XTE}$ buffer at $-20^{\circ} \mathrm{C}$ until PCR amplification.

\subsection{Microsatellite analysis}

The polymorphic SSR markers used in this study are listed in Table 2. All PCR amplifications were performed in thermal cycler (BioRad C1000, USA). The PCR reactions were performed in $25 \mu$ total volume, containing $0.70 \mu \mathrm{l}$ of $0.2 \mathrm{mM}$ dNTPs, $3 \mu \mathrm{l}$ of $2.5 \mathrm{mM} \mathrm{MgCl}_{2}, 4 \mu \mathrm{l}$ of $10 \times$ Taq buffer, $0.2 \mu 1$ of Taq DNA polymerase (Sigma Aldrich, 5 units $/ \mu 1), 1 \mu$ of each primer ( $50 \mathrm{ng} / \mu 1$, Sigma Aldrich, Ge rmany), 20ng of template DNA $(5 \mu \mathrm{l})$ and remaining $\mathrm{dH}_{2} \mathrm{O}$.

Table 2. Summary statistics of Felis Catus SSR markers

\begin{tabular}{|c|l|c|c|}
\hline Microsatellite primers & \multicolumn{1}{|c|}{ Primer sequence ('5-3') } & $\begin{array}{c}\text { Expected } \\
\text { Size Range (bp) }\end{array}$ & EH \\
\hline FCA069 - CT1 & $\begin{array}{l}\text { F-ATCACTCATGCACGAATGC } \\
\text { R-ATTTAACGTTAGGCTTTTTGC }\end{array}$ & $108-118$ & 0.62 \\
\hline FCA075 - CT2 & $\begin{array}{l}\text { F-ATGCTAATCAGTGGCATTTGG } \\
\text { R-GAACAAAAATTCCAGACGTGC }\end{array}$ & $103-143$ & 0.67 \\
\hline FCA105 - CT3 & $\begin{array}{l}\text {-TTGACCCTCATACCTTCTTTGG } \\
\text { R-TGGGAGAATAAATTTGCAAAGC }\end{array}$ & $189-197$ & 0.71 \\
\hline FCA149 - CT4 & $\begin{array}{l}\text {-CCTATCAAAGTTCTCACCAAATCA } \\
\text { R-GTCTCACCATGTGTGGGATG }\end{array}$ & $124-132$ & 0.71 \\
\hline FCA220 - CT5 & $\begin{array}{l}\text {-CGATGGAAATTGTATCCATG } \\
\text { R-GAATGAAGGCAGTCACAAACTG }\end{array}$ & $214-222$ & 0.51 \\
\hline
\end{tabular}

Note: EH= Expected heterozygosity [7].

\subsection{Polymerase Chain Reaction (PCR).}

The PCR protocol included two phases, starting with an initial denaturation phase at $95^{\circ} \mathrm{C}$ for $2 \mathrm{~min}$. The first phase was a touchdown (TD) PCR profile with 20 cycles, starting with $95^{\circ} \mathrm{C}$ denaturation for 30 s, where the annealing temperature was reduced by $-0.7^{\circ} \mathrm{C}$ per cycle for $45 \mathrm{~s}$, followed by the extension stage at $72^{\circ} \mathrm{C}$ for $1 \mathrm{~min}$. The second PCR phase with 15 cycles in total, included $95^{\circ} \mathrm{C}$ denaturation for $30 \mathrm{~s}$, the annealing temperature for each primer was $55^{\circ} \mathrm{C}$, extension at $72^{\circ} \mathrm{C}$ for $30 \mathrm{~s}$. The PCR protocol was completed with the final extension step at $72^{\circ} \mathrm{C}$ for $5 \mathrm{~min}$. The PCR products were separated on $2 \%$ agarose gel, stained with Ethidium bromide $(10 \mathrm{mg} / \mathrm{mL})$, for 60 minutes at $80 \mathrm{~V}$ in $1 \mathrm{x}$ TE buffer. The DNA fragments were photographed and documented using a Gel documentation system (Chemi Doc XRS System, BioRad, USA).

\subsection{Data analysis}

In the analyzed microsatellite loci, the frequency of alleles in each category through all accessions were scored as either present (1) or absent (0). Initially, the potential of all the markers for estimating genetic variability was examined by measuring the marker informativeness through the counting of bands. Additionally, the primer banding characteristics such as number of scored bands (NTB), number of polymorphic bands (NPB) and percentage of polymorphic bands (PPB) were obtained. The discriminatory power of each SSR marker was determined by calculating the polymorphic information content (PIC) and heterozygosity $(\mathrm{H})$ using the online PICcalc program [8].The PIC, H, marker index (MI), the effective multiplex ratio (EMR), the resolving power (RP), were calculated according Karić et.al 2019 [9]. 
A dendrogram was constructed by using hierarchical clustering with UPGMA (Unweighted pair group method with arithmetic mean) analysis, where the genetic distance was measured based on Jaccard's dissimilarity index [10]. The resulting tree was bootstrapped with 1000 replicates to obtain the best confidence [11].For the complete data analysis, the DARwin 6.0.13 software was used [12].

\section{Results}

Three microsatellites markers (CT1, CT4 and CT5) resulted in a total of 10 bands (Table 1), whereas CT2 and CT3 didn't result in any fragments and therefore excluded from further analysis. All the markers showed clear and observable bands (Figure 2). The average PIC values of all primers of SSR analysis were 0.666 The highest observed PIC value was 0.87 with CT1 and the lowest with CT5 primer. The average heterozygosity $(\mathrm{H})$ value of the whole marker set was 0.69 . These values ranged from 0.54 to 0.87 .

Table 3. Genetic diversity estimators for each primer

\begin{tabular}{|l|c|c|c|c|c|c|c|c|c|}
\hline PRIMER & $H$ & $P I C$ & $N S B$ & $N P B$ & $N M B$ & $P P B \%$ & $E M R$ & $M I$ & $R P$ \\
\hline CT1 & 0.89 & 0.87 & 4 & 1 & 3 & 25 & 0.4 & 0.35 & 15.3 \\
\hline CT4 & 0.63 & 0.6 & 2 & 0 & 2 & 0 & 0.0 & 0.0 & 5.5 \\
\hline CT5 & 0.54 & 0.5 & 1 & 0 & 1 & 0 & 0.0 & 0.0 & 5 \\
\hline Mean & 0.69 & 0.66 & 3 & 2.5 & 0.5 & 77.1 & 0.14 & 0.12 & 8.6 \\
\hline
\end{tabular}

H: Heterozygosity; PIC: Polymorphism information contents; NSB: Number of scored band; NPB: Number of polymorphic band;

NMB: Monomorphic bands; PPB: Percentage of polymorphic band; EMR: Effective multiplex ratio; MI: Marker index; RP: Resolving power

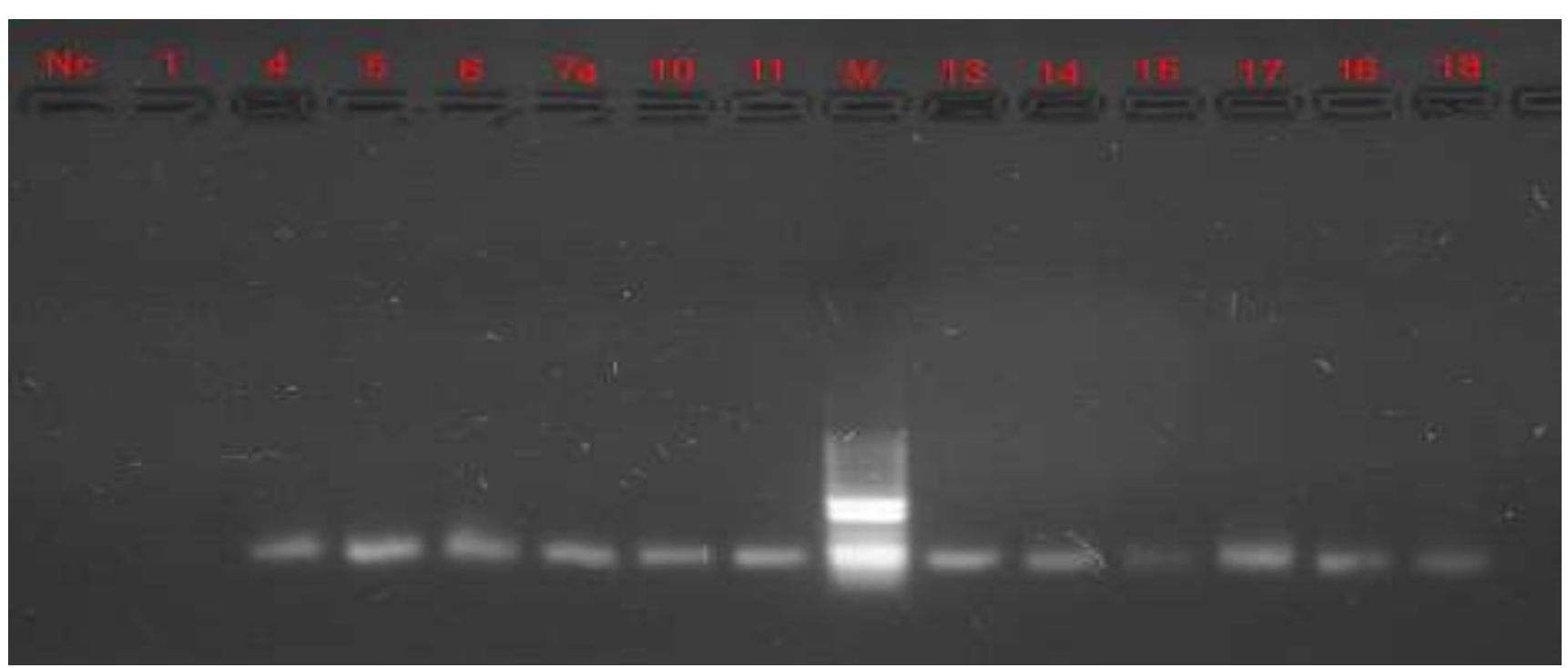

Figure 2: Example of microsatellite profile of different genotypes generated by primer CT1 on $2 \%$ agarose gel. From left to right cat varieties, Nc: Negative control, M (Direct Load ${ }^{\text {TM }}$ PCR 50 bp Ladder

Only one primer resulted in $25 \%$ polymorphic bands (CT1), while the other markers were not polymorphic at all. The effective multiplex ratio (EMR) results for this study showed the that highest value is 0.4 in primer CT1, and the lowest effective multiplex ratio is observed in primers CT4 and CT5 with 0 value, an average EMR of 0.14 per primer. To determine the general usefulness of the system of primers used, the MI (marker index) for each SSR primer was calculated as a product of polymorphic information content and effective multiplex ratio [13]. Highest MI (0.35) was for CT1 and the lowest (0) was for CT4 and CT5.

Resolving power shows the ability of the most informative primers to differentiate between the genotypes assessed [14]. The highest RP value was 15,3, observed with CT1 and the lowest was 5, with primer CT5. 
The phylogenetic dendogram was obtained from 3 SSR markers, revealed by the UPGMA method. The branch lengths are based on the distance values computed using Jaccard's coefficient in Darwin software. The resulted, rooted Phylogenetic UPGMA tree is made of three groups (A, B and C). Group A is divided into two main sub clusters, first out-group with 4 cats- domestic and known breeds ( Žućo, Crna, Pipi and Sivi) and the second sub- group in cluster A, is represented by Mimica cat. These subgroups in cluster A all are sharing common ancestor, but clearly separated from cluster B (made up domestic ESH cats and the subject). The B cluster is made of two sub-clusters, lower Vitany, Subject and Mišur and the upper sub cluster made of Žuti, Cuni, Cuki and Merdanova. The last third group $\mathrm{C}$, is made of lone breed, the Crni cat.

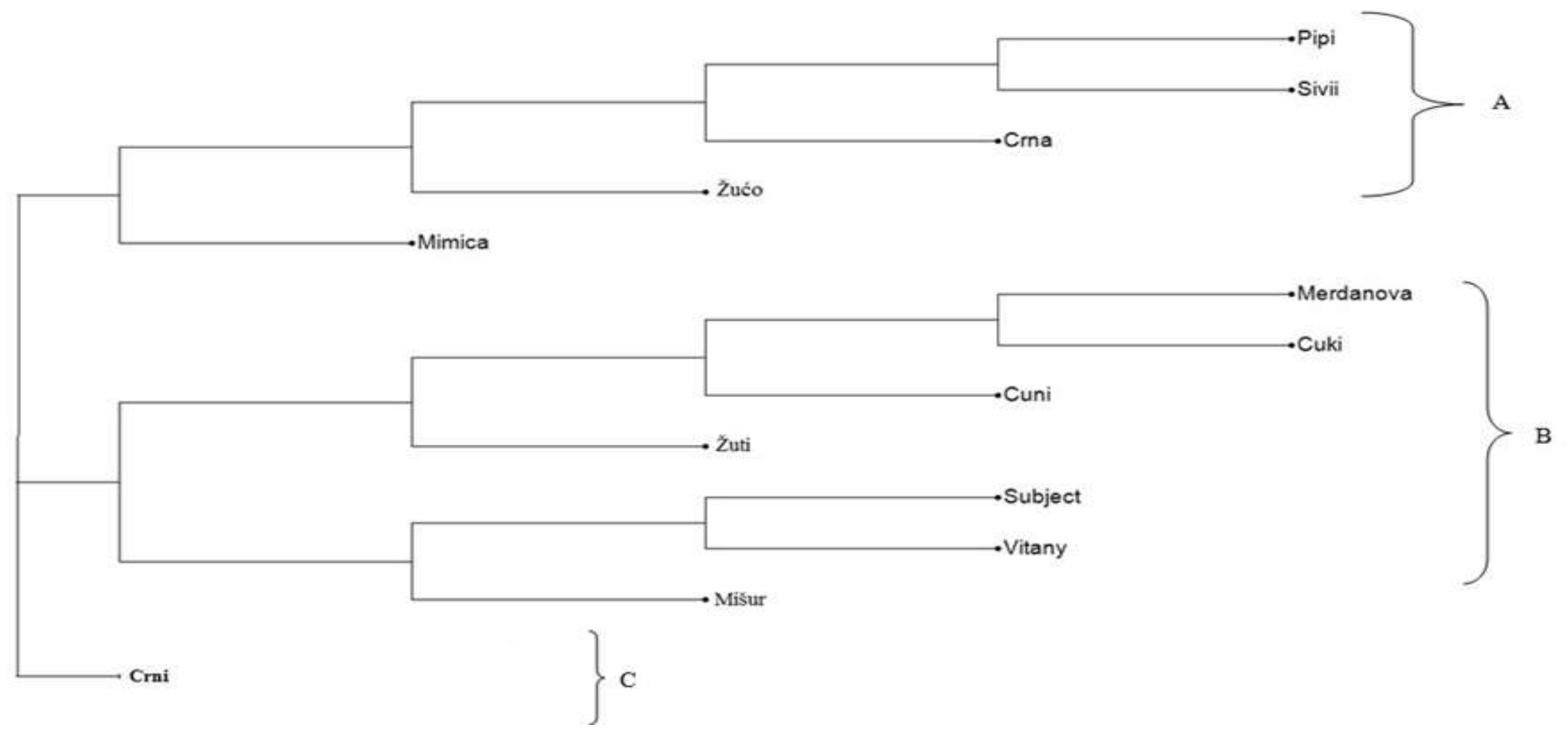

Figure 5: UPGMA Dendogram of the analyzed Cat varieties 


\section{Discussion}

The DNA isolation was not successful in the beginning. The problem lies in the sample storage. Most of these samples were collected 1 to 2 months before the DNA isolation. The buccal mucosa samples were stored in +4 , however, due to improper storage and handling, some samples dried out, which was not sufficient for a successful DNA isolation, where only 13 out of 20 samples were isolated, so samples 2, 3,8,9,12, 19 and 20 were not generically assessed.

Subject and ESH cats have very different morphological appearance, which implies that subjects evolved from a different breed. However, according our Phylogenetic tree, it is genetically closest to the ESH cat- Vitany breed (see figure 5). These results are not representative, since this study involved only 3 genetic markers and out of these 3, two genetic markers CT4 and CT5 did not show to have the required marker impact for genetic diversity analysis. The dendrogram generated using UPGMA cluster analysis grouped 12 genotypes into three clusters. Genetic variation was analyzed using 3 SSRs loci resulted in a total of 7 alleles, out of which 2 bands $(25.6 \%)$ were polymorphic. It is reported that high diversity of locus is demonstrated in PIC values greater than 0.5 [15]. In our study, all samples had more than 0.6 polymorphic information content. The PIC value will be almost zero if there is no allelic variation and it can reach a max of 1.0 if a genotype has only new allele [16]. However, the primers CT1 and CT5 showed higher PIC values if compared to a similar study done with the same primers [4].

The average value of the total heterozygosity $(\mathrm{H})$ in our results was 0.67 , whereas CT1 and CT5 showed the expected and higher heterozygosity $(\mathrm{H})$ values than previous studies have demonstrated (see table 3 ), where the same primes showed 0.8 and 0.44 values, respectively [4]. In this regards, only CT1 primer was informative, and sufficient for distinguishing the polymorphic rate of loci. Thereof, the RP values and all other maker index values (shown in table 9) are in line with PIC and H data. According this result, CT1 with 15.3 RP is the most informative primer for distinguishing the analyzed cat breed. However, the resolving power provides no information on the ability of a primer to reflect the genetic or taxonomic relationships of a group of genotypes under the study [14].

These results indicate that only CT1 marker is sufficient for the analysis of both interspecific and intraspecific genetic diversity, as a potent marker for fine dissection of the intraspecific relationship in cat breeds, as shown in the previous study [4].

\section{Conclusion}

To conclude, this study tried to genetically characterize the domestic and one unknown cat breed by using three genetic primers. Based on all marker impact parameters (shown in table 9) only one marker (CT1) showed to be polymorphic characteristics for sufficient genetic characterization. Accordingly, the subject cat showed to share common ancestor with Vitany breed. For a more precise and significantly effective study, minimum 10 primers should be used for a better genetic characterization, to show a clearer differentiation among the cats. This study presents the first genetic analysis study on cat varieties from Bosnia and Herzegovina.

\section{References}

[1] Felis catus Linnaeus, 1758 in Döring M . English "Wikipedia - Species Pages". Wikimedia Foundation. Checklist dataset https://doi.org/10.15468/c3kkgh , accessed via GBIF.org on 2021-0210, 2021.

[2] Sparks, Andrew H., Katherine Rogers, William E. Henley, Danielle A. Gunn-Moore, Julia M. May, Timothy J. Gruffydd-Jones, and Claire Bessant. "A questionnaire-based study of gestation, parturition and neonatal mortality in pedigree breeding cats in the UK." Journal of feline medicine and surgery 8 , no.3, pp. 145-157, 2006

[3] European Shorthair Cat Breed: "Facts and Personality Traits " .Hillspets, accessed via https://www.hillspet.com/cat-care/cat-breeds/european-shorthair on 2021-02-10, 2021.

[4] Eizirik, Eduardo, et al. "Defining and mapping mammalian coat pattern genes: multiple genomic regions implicated in domestic cat stripes and spots." Genetics 184, pp. 267-275, 2010 . 
[5] Gross, Dominik, and René H. Tolba. "Ethics in animal-based research." European Surgical Research 55.1-2,pp. 43-57, 2015

[6] Aidar, Marisi, and Sergio Roberto Peres Line. "A simple and cost-effective protocol for DNA isolation from buccal epithelial cells." Brazilian dental journal 18.2,pp.148-152, 2007.

[7] Menotti-Raymond, Marilyn, et al. "A genetic linkage map of microsatellites in the domestic cat (Felis catus)." Genomics 57.1,pp.9-23, 1999.

[8] Nagy, Sándor, et al. "PICcalc: an online program to calculate polymorphic information content for molecular genetic studies." Biochemical genetics 50.9-10 ,pp. 670-672, 2012.

[9] Karić L, Golzardi, M., Glamoclija, P., and Sutkovic, J., "Genetic diversity assessment of Allium cepa L. cultivars from Bosnia and Herzegovina using SSR makers", Genetics and Molecular Research, vol. 17, no. 1, 2018. DOI http://dx.doi.org/10.4238/gmr16039870

[10] Real, Raimundo, and Juan M. Vargas. "The probabilistic basis of Jaccard's index of similarity." Systematic biology 45.3 ,pp.380-385, 1996.

[11] Felsenstein, Joseph. "Confidence limits on phylogenies: an approach using the bootstrap." evolution 39.4,pp .783-791,1985.

[12] Perrier X, Jacquemoud-Collet JP, Darwin (2006). http://darwin.cirad.fr/.

[13] Powell, Wayne, Michele Morgante, Chaz Andre, Michael Hanafey, Julie Vogel, Scott Tingey, and Antoni Rafalski. "The comparison of RFLP, RAPD, AFLP and SSR (microsatellite) markers for germplasm analysis." Molecular breeding 2, no. 3 (1996): 225-238.

[14] Prevost, A., and M. J. Wilkinson. "A new system of comparing PCR primers applied to ISSR fingerprinting of potato cultivars." Theoretical and applied Genetics 98, no. 1 (1999): 107-112.

[15] Alhasnawi, Arshad Naji, Amar Mousa Mandal, and Haider Mahmooed Jasim. "Using DNA fingerprinting to detect the genetic relationships in Acacia by inter-simple sequence repeat markers." $J$ Pure Appl Microbiol 13.1,pp.281-288, 2019.

[16] Guo X, Elston R. "Linkage information ontent of polymorphic genetic markers ". Human heredity. 49:2:pp.112-118, 2009. https://doi.org/10.1159/000022855 